\title{
Sgc8-c Aptamer as a Potential Theranostic Agent for Hemato-Oncological Malignancies
}

\author{
Estefanía Sicco, Jessica Baez, ${ }^{1}$ Manuel Ibarra, ${ }^{2}$ Marcelo Fernández, ${ }^{3}$ \\ Pablo Cabral, ${ }^{1}$ María Moreno, ${ }^{4}$ Hugo Cerecetto, ${ }^{1}$ and Victoria Calzada ${ }^{1}$
}

\begin{abstract}
Background: Aptamers represent an emerging class of oligonucleotides that have the ability to bind ligands with high affinity. Sgc8-c aptamer recognizes PTK7, a member of the catalytically defective receptor protein tyrosine kinase family that is upregulated in various cancers, including hemato-oncological malignancies. Herein, an Sgc8-c-NOTA-radiolabeled probe was prepared for theranostic purpose.

Materials and Methods: In this work, an Sgc8-c-radiolabeled probe against PTK7 was prepared, and biological evaluations-pharmacokinetic studies, biodistribution analysis, and in vivo molecular imaging - were performed. To obtain the radiolabeled probe, a modified 5'-amino-derivative of the Sgc8-c aptamer was bound to the metal chelator NOTA, and subsequently labeled with ${ }^{67} \mathrm{Ga}$ with high yield and radiochemical purity. The precursor, Sgc8-c-NOTA, the radio probe Sgc8-c-NOTA- ${ }^{67} \mathrm{Ga}$, and its nonradioactive complex, Sgc8-cNOTA- ${ }^{69 / 71} \mathrm{Ga}$, were purified by reverse-phase high-performance liquid chromatography and characterized by electrospray ionization mass spectrometry. The binding ability of Sgc8-c-NOTA- ${ }^{67} \mathrm{Ga}$ was studied in vitro against purified PTK7 receptor. In addition, the binding was also evidenced against the hemato-oncological A20 cell line, derived from B lymphocytes, and the corresponding A20-green fluorescent protein (GFP)-transfected cells. The proof of concept was performed on A20-GFP tumor-bearing mice, in which the biodistribution of the radiolabeled probe was evaluated through imaging, using $\mathrm{X}$-ray, fluorescence, and $\gamma$ modalities. The specific uptake of the probe was confirmed by blocking with the Sgc8-c aptamer in an in vivo competition assay.

Results: The biodistribution results showed considerable uptake in tumor since $2 \mathrm{~h}$, with highest at $48 \mathrm{~h}$ postinjection. However, the blood and muscle ID/g (injected dose per gram of tissue) activities were decreasing with time and tumor/no-target ratios increasing to 20 at $24 \mathrm{~h}$ postinjection. These results are consistent with the in vivo images.
\end{abstract}

Conclusions: This study supports the utility of Sgc8-c-NOTA radiolabeled as a theranostic agent.

Keywords: aptamer, PTK7, lymphoma, theranostic

\section{Introduction}

A ptamers are small single-stranded oligonucleotides highly selective for different targets. ${ }^{1}$ They can be artificially selected by the Systematic Evolution of Ligands by Exponential Enrichment (SELEX) against a large variety of targets from small molecules to live cells. ${ }^{2-5}$ Thus, a wide range of biotechnology purposes have been developed such as biosensors, ${ }^{6}$ drug delivery systems, ${ }^{7}$ flow cytometry, imaging, diagnostics, ${ }^{8}$ and therapeuthics. ${ }^{9}$ Aptamers are considered promising tools safe for in vivo use, as they have been reported to possess essential clinical aspects, such as

\footnotetext{
${ }^{1}$ Departamento de Radiofarmacia, Centro de Investigaciones Nucleares, Facultad de Ciencias, Universidad de la República, Montevideo, Uruguay.

${ }^{2}$ Departamento de Ciencias Farmaceuticas, Facultad de Química, Universidad de la República, Montevideo, Uruguay.

${ }^{3}$ Laboratorio de Experimentacion Animal, Centro de Investigaciones Nucleares, Facultad de Ciencias, Universidad de la República, Montevideo, Uruguay.

${ }^{4}$ Departamento de Desarrollo Biotecnológico, Instituto de Higiene, Facultad de Medicina, Universidad de la República, Montevideo, Uruguay.
}

Address correspondence to: Victoria Calzada; Departamento de Radiofarmacia, Centro de Investigaciones Nucleares, Facultad de Ciencias, Universidad de la República; Mataojo 2055, Montevideo 11200, Uruguay

E-mail: hcerecetto@cin.edu.uy 
nonimmunogenic and nontoxic properties. ${ }^{10}$ However, aptamers have been shown to be rapidly degraded by nucleases, and for this reason, different chemical modifications have been reported for prestabilization and protection. ${ }^{11}$ Moreover, chemical modifications could modulate fundamental aspects like residence time circulation and tissue distribution. $^{12}$

Sgc8-c is a $41 \mathrm{nb}$ DNA aptamer selected with cellSELEX by Tan et al. ${ }^{13}$ This aptamer recognizes PTK7, a member of the catalytically defective receptor protein tyrosine kinase family, which is overexpressed in several cancer cell lines. Patient screenings have demonstrated that PTK7 is expressed in hemato-oncological malignancies like acute myeloid leukemia, biphenotypic acute leukemia, and myeloproliferative syndromes. ${ }^{14}$ This expression profile makes PTK7 a tumoral marker candidate for developing diagnostic and therapeutic strategies.

In this sense, the authors previously demonstrated the potential of Scg8-c as a molecular imaging agent, for lymphoma and melanoma pathologies, using a fluorescent and two radiolabeled probes. Lymphomas represent a diverse group of neoplastic disorders of lymphocytes and are very frequent, requiring imaging to help the clinician in the therapies. ${ }^{15}$ Within the radiolabeled probes, they obtained promising preliminary results with a ${ }^{67} \mathrm{Ga}$-DOTA Sgc8-c derivative, reporting good tumoral biodistribution and imaging results. ${ }^{16}$ This radio probe showed increased liver uptake and bone uptake over time, consistent with hepatobiliary metabolism and some potential degree of instability of coordinated gallium. In addition, tumor/blood and tumor/muscle ratios were higher than 6 , increasing with time. This in vivo behavior could be modified using a different $\mathrm{Ga}$ chelator, such as NOTA (1,4,7-triazacyclononane-1,4,7triacetic acid). ${ }^{17}$ To verify the chelator influence, herein, they report on the synthesis of a second series of ${ }^{67} \mathrm{Ga}$ radiolabeled Sgc8-c, using 4-\{[1,4,7-tri(carboxymethyl)1,4,7-triazacyclononane-2-yl]methyl\}phenylthiourea as a connector/chelator and its evaluation in vitro and in vivo. In vitro studies included the determination of $\log \mathrm{D}, \mathrm{PTK} 7-$ and cell-binding abilities. In vivo experiments included determination of pharmacokinetic behavior, biodistribution, and imaging using a pre-established model of hematotumor-bearing animals. ${ }^{18}$

\section{Materials and Methods}

\section{General methods}

Chemistry. Pure commercially available materials, reagents, and solvents were used without further purification. 5'-Aminohexyl-modified Sgc8-c aptamer ( $13 \mathrm{kDa}, \mathrm{Sgc} 8-\mathrm{c}-$ $\mathrm{NH}_{2}$ ) was purchased from IDT Technologies (Integrated DNA Technologies, Coralville, IO). All buffers and aqueous solutions were prepared using metal-free MilliQ water. As previously described in detail, ${ }^{19}$ advance of the reaction and the product quality were followed by reverse-phase highperformance liquid chromatography (RP-HPLC; Agilent 1200 Series Infinity Star, Santa Clara, CA) with a $5 \mu \mathrm{m} \mathrm{C-18}$ Kinetex column (Phenomenex). For each run, an aqueous solution was used, consisting of triethylamine $(50 \mathrm{mM}, \mathrm{pH}$ 7.5)/5\% acetonitrile (solvent $\mathrm{A}$ ) and methanol (solvent $\mathrm{B}$ ), at a flow of rate $1 \mathrm{~mL} / \mathrm{min}$, and performed using a gradient of A:B (90:10) to A:B (40:60) over $30 \mathrm{~min}$ (ultraviolet [UV] or $\gamma$ detections). Purifications of the precursor and gallium complexes were performed with the same solvent gradient. ${ }^{16,18,19}$

Biology. The A20 cell line derived from mouse B lymphocytes of a naturally occurring reticulum cell sarcoma in an old BALB/cAnN mouse was used and obtained from the American Type Culture Collection (Manassas, VA). In addition, A20/green fluorescent protein (GFP)-transfected cells were constructed in the authors' laboratory based on the method described by Kalil et al. ${ }^{20}$ Briefly, $2 \times 10^{5}$ cells were transfected with commercial pEGFP-N1 plasmid (Clontech Lab, Palo Alto, CA) (500 ng) in Dulbecco's modified Eagle's medium $(50 \mu \mathrm{L})$ with lipofectamine (Life Technologies, Carlsbad, CA) for $48 \mathrm{~h}$. Afterward, the culture media were changed and the cells were grown in the presence of geneticin $(200 \mu \mathrm{g} / \mathrm{mL})$ (Sigma-Aldrich, St. Louis, MO) for 1 month. The highest fluorescent cells $(0.4 \%)$ were selected using flow cytometry (MoFlo; Beckman Coulter, Pasadena, CA). A20 and A20/GFP cells were grown in suspension in RPMI-1640 media (Sigma-Aldrich) supplemented with 10\% (v/v) fetal bovine serum (FBS), $2 \mathrm{mM}$ L-glutamine, and $0.05 \mathrm{mM} \beta$-mercaptoethanol (Sigma-Aldrich). All cell lines were cultured at $37^{\circ} \mathrm{C}$ with $5 \%(\mathrm{v} / \mathrm{v}) \mathrm{CO}_{2}$.

Confirmation of PTK7 expression by A20/GFP cells using flow cytometry. Briefly, expression levels were evaluated by staining the cells with the anti-PTK7-PE (phycoerythrin) antibody (Clone Type: Polyclonal, Catalog No: 033359-PE; United States Biological) and detected in a FACS Canto II flow cytometer (BD Biosciences, San Diego, CA). Data were analyzed using FACS Diva and FlowJo software. The human acute lymphoblastic leukemia cell line CCRF-CEM (ATCC, Manassas, VA) was used as a positive control, ${ }^{14}$ while the malignant glioma derived cell line U-87 MG (HTB-14RTM; ATCC) was used as a negative control in this study. ${ }^{21}$

Animals. All protocols for animal experimentation were carried out in accordance with guidelines for the care and use of animals and the procedures were approved by the University's Ethical Committee for Animal Experimentation, Uruguay, to whom this project was previously submitted (Approval No: 240011-001891-17). Animals purchased from DILAVE (Montevideo, Uruguay) were housed in boxes with up to five animals and kept with water and food ad libitum, with $14 \mathrm{~h}$ of light and $10 \mathrm{~h}$ of darkness. They were monitored daily, registering the animals' behavior. In the case of animals bearing tumors, the tumors were palpated to record their presence, location, and volume, where they reached a maximum diameter of $5 \mathrm{~mm}$. Isoflurane was used for anesthesia. At the end of the experiments, all animals were sacrificed by cervical dislocation.

\section{Synthesis of Sgc8-c-NOTA}

Sgc8-c-NOTA was prepared according to the authors' previous report. ${ }^{19}$

\section{Sgc8-c-NOTA gallium coordination (Sgc8-c-NOTA-Ga)}

Sgc8-c-NOTA was coordinated with stable gallium based on the following: Sgc8-c-NOTA $(20 \mu \mathrm{g}, 1.5 \mathrm{nmol})$ was dissolved in ammonium acetate buffer $(0.1 \mathrm{M}, \mathrm{pH}=6.0,300 \mu \mathrm{L})$ and ${ }^{69 / 71} \mathrm{GaCl}_{3}$ in excess (427128; Sigma-Aldrich) was added. 
The mixture was stirred at $60^{\circ} \mathrm{C}$ for $30 \mathrm{~min}$, and the reaction was followed using RP-HPLC. The new product (checked at $\lambda=260 \mathrm{~nm}, t_{\mathrm{R}}=23.6 \mathrm{~min}$ ) was collected from the RP-HPLC analysis and the structure was confirmed by electrospray ionization mass spectrometry (ESI-MS; IDT Technologies). Yield: $50 \%$. ESI-MS, Sgc8-c-NOTA- ${ }^{69 / 71} \mathrm{Ga}$, found: 13327.1 Da (M-2H); expected: $13329.5 \mathrm{Da}$. The same synthetic procedure was performed using radioactive ${ }^{67} \mathrm{GaCl}_{3}$ solution (Tecnonuclear, Buenos Aires, Argentina), using 30.7 MBq per each $0.4 \mathrm{nmol}$ of Sgc8-c-NOTA in a final volume of $1000 \mu \mathrm{L}$ with ammonium acetate buffer $(0.1 \mathrm{M}, \mathrm{pH}=6.0)$. The RPHPLC radioactive peak had a $t_{\mathrm{R}}=23.8 \mathrm{~min}$. The radiolabeling yield and radiochemical purity were monitored using RPHPLC with a $\gamma$ detector comparing with retention time of the UV signal of the precursor and cold complex. Radiochemical purity: $97.2 \%$. The final specific activity, calculated by dividing the obtained radioactivity by the mass unit of Sgc8-cNOTA used for the reaction, was $76.7 \mathrm{MBq} / \mathrm{nmol}$.

\section{In vitro characterization}

Media reaction stability studies with time and temperature. Sgc8-c-NOTA- ${ }^{67} \mathrm{Ga}(0.08 \mathrm{nmol})$ was maintained in media reaction $(400 \mu \mathrm{L})$ at room temperature $\left(25^{\circ} \mathrm{C}\right)$ for $48 \mathrm{~h}$. After 24 and $48 \mathrm{~h}$, aliquots were studied by RP-HPLC. On the other hand, Sgc8-c-NOTA- ${ }^{67} \mathrm{Ga}(0.08 \mathrm{nmol})$ was incubated in media reaction $(400 \mu \mathrm{L})$ at different temperatures $\left(25^{\circ} \mathrm{C}, 37^{\circ} \mathrm{C}, 45^{\circ} \mathrm{C}, 60^{\circ} \mathrm{C}\right.$, and $\left.75^{\circ} \mathrm{C}\right)$, for $30 \mathrm{~min}$. After the time points, aliquots from samples at each temperature were studied by radio-RP-HPLC.

Serum fetal bovine stability. The probe $(0.08 \mathrm{nmol})$ was incubated with FBS $(200 \mu \mathrm{L})$ at $37^{\circ} \mathrm{C}$ for $24 \mathrm{~h}$. After $30 \mathrm{~min}$, and 2 and $24 \mathrm{~h}$, the mixture was filtered by 10 and $30 \mathrm{kDa}$ microcon ${ }^{\circledR}$ centrifugal filters and studied by RP-HPLC.

Distribution coefficient $(\log D)$. To determine the distribution of Sgc8-c-NOTA- ${ }^{67} \mathrm{Ga}$ between $n$-octanol and phosphate buffered saline (PBS), $\mathrm{pH}=7.5$, aliquots of the probe $(0.075 \mathrm{nmol})$ were diluted in $500 \mu \mathrm{L}$ of PBS, and after adding $500 \mu \mathrm{L}$ of $n$-octanol, the mixture was centrifugated at $13,000 \mathrm{rpm}$ for $10 \mathrm{~min}$ at room temperature. Subsequently, aliquots $(200 \mu \mathrm{L})$ of both layers were collected and measured in a $\gamma$ counter (PC-RIA MAS; Stratec), followed by the $\log \mathrm{D}$ calculation $(n=4$, eight replicates).

\section{Bioactivity experiments}

The maximum binding capacity assay was performed following the Lindmo method. ${ }^{22}$ Different amounts of pure receptor PTK7-1 (TP700163; Origene, MD) were prepared in triplicate in $2 \mathrm{~mL}$ of $\mathrm{PBS}(\mathrm{pH}=7.5)$ to give final concentrations of $9 \times 10^{-3}, 18 \times 10^{-3}, 35 \times 10^{-3}, 71 \times 10^{-3}$, $0.140,0.575,1.150$, and $4.600 \mathrm{nM}$, subsequently adsorbed in Nunc tubes (Brandt ${ }^{\circledR}$ ). After incubating for $24 \mathrm{~h}$ at $4^{\circ} \mathrm{C}$, with continuous gentle orbital shaking, the tubes were washed two times with PBS. Finally, 100,000 cpm of Sgc8-cNOTA- ${ }^{67} \mathrm{Ga}$ was incubated for $2 \mathrm{~h}$ at room temperature in the darkness and with continuous orbital shaking. The tubes were washed two times with PBS and the radioactivity was quantified using a $\gamma$ counter (PC-RIA MAS; Stratec). Nonspecific binding was determined by incubating Sgc8-cNOTA- ${ }^{67} \mathrm{Ga}$ without receptor.
Cell binding was studied in A20 and A20/GFP cell lines. All cells were washed twice by gentle centrifugation with sterile PBS $(\mathrm{pH}=7.4)$ and $1.0 \times 10^{6}$ cells were re-suspended in PBS (final volume of $1 \mathrm{~mL}$ ) and incubated with 100,000 $\mathrm{cpm}$ of $\mathrm{Sgc} 8 \mathrm{c}-\mathrm{NOTA}-{ }^{67} \mathrm{Ga}$. The probe was incubated for $0.5,2$, and $4 \mathrm{~h}$ at $37^{\circ} \mathrm{C}$. Cells were washed twice with PBS and radioactivity in the pellets was quantified in a $\gamma$ counter (PC-RIA MAS; Stratec). Competition binding assays were performed with excess $\mathrm{Sgc} 8-\mathrm{c}-\mathrm{NH}_{2}(5 \mu \mathrm{g}, 0.4 \mathrm{nmol})$ incubated for $30 \mathrm{~min}$ at $37^{\circ} \mathrm{C}$. After incubation with $\mathrm{Sgc} 8-\mathrm{c}-\mathrm{NH}_{2}$, the cells were washed with PBS and incubated with 100,000 $\mathrm{cpm}$ of Sgc8c-NOTA- ${ }^{67} \mathrm{Ga}$. The probe was incubated for $2 \mathrm{~h}$ at $37^{\circ} \mathrm{C}$. The cells were washed twice with PBS and radioactivity in the pellets was quantified as described above.

\section{Pharmacokinetic studies}

The pharmacokinetic profile was assessed by administering an intravenous (IV) bolus of individual doses of 5.5-9.2 MBq of Sgc8c-NOTA- ${ }^{67} \mathrm{Ga}$ into the tail vein of female Wistar rats, weighting 200-250 g $(n=3)$. Blood samples were collected in a capillary tube from the orbital sinus at $0.25,0.5,1,2,4,6,18,24$, and $48 \mathrm{~h}$ following probe administration. Samples were weighed and radioactivity was measured in a $\gamma$ counter (PC-RIA MAS; Stratec). Urine and fecal matter were also collected over a 48-h period to assess renal elimination. A compartmental pharmacokinetic analysis was performed for blood and urine data using the software for nonlinear mixed-effects modeling NONMEM 7.4.1. ${ }^{23}$ Model development was conducted based on the Akaike information criteria and goodness-of-fit plots.

\section{In vivo experiments}

Tumor model was generated in female BALB-c mice at 4-6 weeks of age, subcutaneously injected with $1 \times 10^{6}$ A20/ GFP cells resuspended in $200 \mu \mathrm{L}$ of culture media. Formation of tumors was observed 2 weeks later, reaching maximum sizes of $5 \mathrm{~mm}$ of diameter. ${ }^{24}$ No animal died during the study.

Biodistribution. When the tumors were palpable, Sgc8cNOTA- ${ }^{67} \mathrm{Ga}(\sim 1850 \mathrm{kBq})$ was IV administered through the tail vein. At $0.5,2,24,48$, and $72 \mathrm{~h}$ after probe administration, the mice ( $n=5$ per time point) were sacrificed by cervical dislocation and the organs were weighed and measured in a $\gamma$ counter (PC-RIA MAS; Stratec). Radioactivity levels in each tissue were expressed as percentages of the injected dose per gram of tissue $(\% \mathrm{ID} / \mathrm{g})$ and as percentages of injected dose (\%ID). An in vivo blocking experiment was performed in a separate group $(n=5)$, with a previous injection of 100-fold excess of Sgc8-c- $\mathrm{NH}_{2}$ aptamer. A half hour before the injection of the radioactive probe $(\sim 1850 \mathrm{kBq}), 0.5 \mathrm{nmol}$ of $\mathrm{Sgc} 8-\mathrm{c}-\mathrm{NH}_{2}$ was administrated through the tail vein. The mice were sacrificed $2 \mathrm{~h}$ after the radioactive probe injection and measurements were performed as previously described. Tissue decay correction and organ weight correction were applied, and the tumor/blood and tumor/muscle ratios were calculated.

Imaging. When the tumors were palpable, Sgc8cNOTA- ${ }^{67} \mathrm{Ga}(\sim 1850 \mathrm{kBq})$ was IV administered through the tail vein. After 2, 24, 48, and $72 \mathrm{~h}$, the mice were anesthetized 
with isoflurane. Images were taken with the In-Vivo MS FX PRO instrument (Bruker, Billerica), using the X-ray, fluorescence, and $\gamma$ modes (10 min acquisition). After each imaging time point, the mice were sacrificed for organ dissection and imaged and counted separately using the same imaging instrument (In-Vivo MS FX PRO instrument; Bruker). The organ weight correction was applied and the tumor/blood and tumor/muscle ratios were calculated.

\section{Statistical analysis}

Statistical analysis was performed using the Student's $t$-test with $p$-values of significance indicated in each case.

\section{Results}

\section{Radiolabeling}

The synthesis of $\mathrm{Sgc} 8 \mathrm{c}-\mathrm{NOTA}-{ }^{67} \mathrm{Ga}$ was a one-step procedure from the precursor Sgc8-c-NOTA ${ }^{19}$ and ${ }^{67} \mathrm{GaCl}_{3}$, under optimized conditions (Fig. 1). Aptamers are chemically stable in neutral conditions and low temperatures and are soluble in nonorganic solvents. For these reasons, several radiolabeling conditions $(\mathrm{pH} / \mathrm{buffer}$ of reaction, temperature, time, Sgc8-c-NOTA concentration, and radioactivity) were studied to produce the desired product with high labeling efficiency, in a short time. In all experiments, the highest purity grade reagents available and metal-free water were used to minimize the effects of metal contaminants interfering with gallium radiolabeling.

At $\mathrm{pH}$ values between 3 and $7, \mathrm{Ga}^{3+}$ forms insoluble $\mathrm{Ga}(\mathrm{OH})_{3}$, but deprotonation of NOTA-carboxylic moieties for better complexation requires $\mathrm{pH}$ higher than 5. Thus, buffer systems with an adequate $\mathrm{pH}$ play a relevant role in the labeling procedure. Within this context, two buffer systems of reaction were studied, sodium acetate $(0.1 \mathrm{M}$, $\mathrm{pH}=5.0)^{25,26}$ and ammonium acetate $(0.1 \mathrm{M}, \mathrm{pH}=5.0),{ }^{16}$ at $37^{\circ} \mathrm{C}$ for $30 \mathrm{~min}$. In sodium acetate buffer, no radiolabeled (rdl) was observed, while a little degree of rdl was evidenced in the ammonium acetate (Supplementary Table S1). Consequently, using ammonium acetate buffer, the authors modified the temperature and time of the reaction, finding that a modest improvement in the rdl was reached working at $60^{\circ} \mathrm{C}$, in accordance with their previous findings, ${ }^{16}$ decreasing the rdl when the reaction was carried out for up to $45 \mathrm{~min}$ (Supplementary Table S1). Due to the very slow gallium incorporation, which was possibly a result of the low $\mathrm{pH}$, they studied two additional $\mathrm{pH}$ conditions, $\mathrm{pH}=5.5$ and $\mathrm{pH}=6.0$, finding that a slight change in the $\mathrm{rdl}$ was observed at $\mathrm{pH}=5.5$ (compare rdl for run 2 in Supplementary Table S1 and run 4 in Supplementary Table S2) with best improvement at the highest $\mathrm{pH}$ studied (run 7 in Supplementary Table S2).

To establish a mild radiolabeling method in ammonium acetate buffer $(0.1 \mathrm{M}, \mathrm{pH}=6.0)$, the complexation reaction was studied at $37^{\circ} \mathrm{C}$ during the time (Supplementary Table S3). This temperature did not result in better condition and as it was observed in other experiments, prolonged times produced rdl diminution (see also run 3 in Supplementary Table S1 and run 5 in Supplementary Table S2). In contrast, the $\mathrm{rdl}$ efficiency at $\mathrm{pH}=6.0,60^{\circ} \mathrm{C}$, and $30-\mathrm{min}$ incubation period was dependent on the molar quantity of the precursor (Supplementary Table S4), increasing from $40 \%$ to $77 \%$ when using 0.08 and $0.4 \mathrm{nmol}$ (corresponding to concentrations of 0.2 and $0.4 \mu \mathrm{M}$ ), respectively, of Sgc8c-NOTA. Finally, rdl was studied as a function of the ${ }^{67} \mathrm{Ga}$ activity (Fig. 2 and Supplementary Table S5). The rdl efficiency was activity dependent, reaching the best value at $30.7 \mathrm{MBq}$ of ${ }^{67} \mathrm{GaCl}_{3}$, with a slight decrease at $37 \mathrm{MBq}$. It showed that higher volumes of the ${ }^{67} \mathrm{Ga}$ solution (Supplementary Table S5) could incorporate higher concentrations of metal cation contaminants, like $\mathrm{Fe}(\mathrm{III})$, decreasing the ${ }^{67} \mathrm{Ga}$ incorporation. ${ }^{27}$

In the optimal condition, $\mathrm{pH}=6.0,60^{\circ} \mathrm{C}, 30 \mathrm{~min}, 0.4 \mu \mathrm{M}$ of Sgc8-c-NOTA, and $30.7 \mathrm{MBq}$ of ${ }^{67} \mathrm{GaCl}_{3}$ (run 19 in Supplementary Table S5), purification of the resulting Sgc8-c-NOTA- ${ }^{67} \mathrm{Ga}$ was not required, because the rdl was over $95 \%$ and the reaction buffer was compatible with biological systems. The identity of Sgc8-c-NOTA- ${ }^{67} \mathrm{Ga}$ was investigated by HPLC and ESI-MS. The HPLC analytical results of the Sgc8-c-NOTA- ${ }^{67} \mathrm{Ga}$ were compared with the same conjugate that was complexed with stable ${ }^{69 / 71} \mathrm{Ga}$ nuclides, using ${ }^{69 / 71} \mathrm{GaCl}_{3}$, under similar conditions at its radioactive counterpart. Figure 3 shows a clear overlap of the corresponding peaks in the UV detector chromatograms of RP-HPLC for the ${ }^{69 / 71} \mathrm{Ga}$-complexed Sgc8-c derivative, Sgc8-c-NOTA- ${ }^{69 / 71} \mathrm{Ga}$, and the ${ }^{67} \mathrm{Ga}$-radiolabeled desired product, demonstrating similar identities. In addition, the identity of the Sgc8-c-NOTA- ${ }^{69 / 71} \mathrm{Ga}$ was confirmed by ESI-MS (Supplementary Fig. S1).

\section{In vitro characterization}

The Sgc8-c-NOTA- ${ }^{67}$ Ga distribution coefficient $(\log \mathrm{D}$, $\mathrm{pH}=7.5$ ) was $-2.41 \pm 0.11$. The results of the stability in media reaction studies showed that the radio probe was stable at room temperature $\left(25^{\circ} \mathrm{C}\right)$ for $48 \mathrm{~h}$ and up to $75^{\circ} \mathrm{C}$

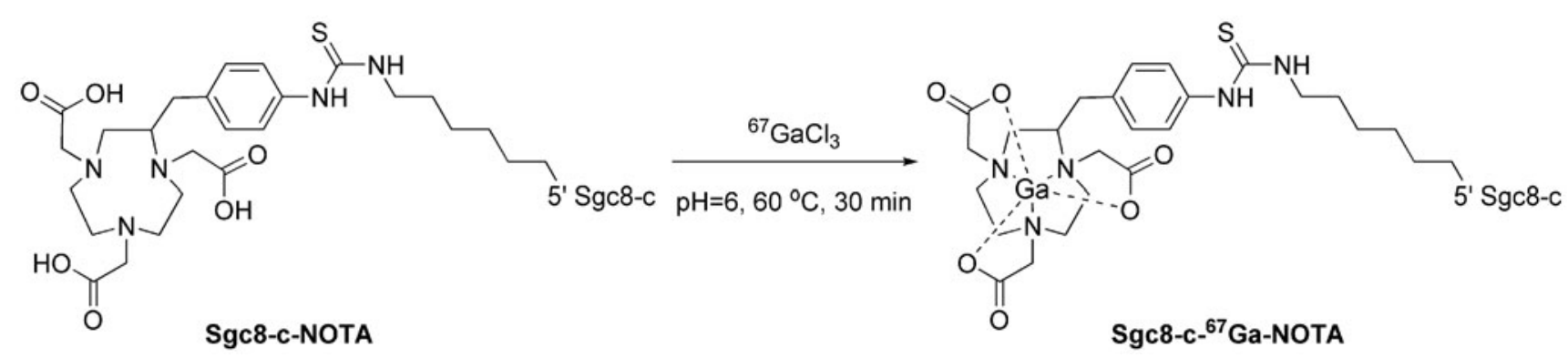

FIG. 1. Reaction scheme for the complexation of ${ }^{67} \mathrm{Ga}^{3+}$ with Sgc8-c-NOTA. 
FIG. 2. Summary of Sgc8c-NOTA Ga-radiolabeled process as function of the different studied variables (shown results were obtained, apart from the variable indicated conditions, at $60^{\circ} \mathrm{C}$ for $\left.30 \mathrm{~min}\right)$. The best condition of complexation was ammonium acetate buffer $(0.1 \mathrm{M}, \mathrm{pH}=6.0)$, $0.4 \mathrm{nmol}$ of Sgc8-c-NOTA, $30.7 \mathrm{MBq}$ of ${ }^{67} \mathrm{GaCl}_{3}$, and at $60^{\circ} \mathrm{C}$ for $30 \mathrm{~min}$ (final volume of reaction $1000 \mu \mathrm{L}$ ).

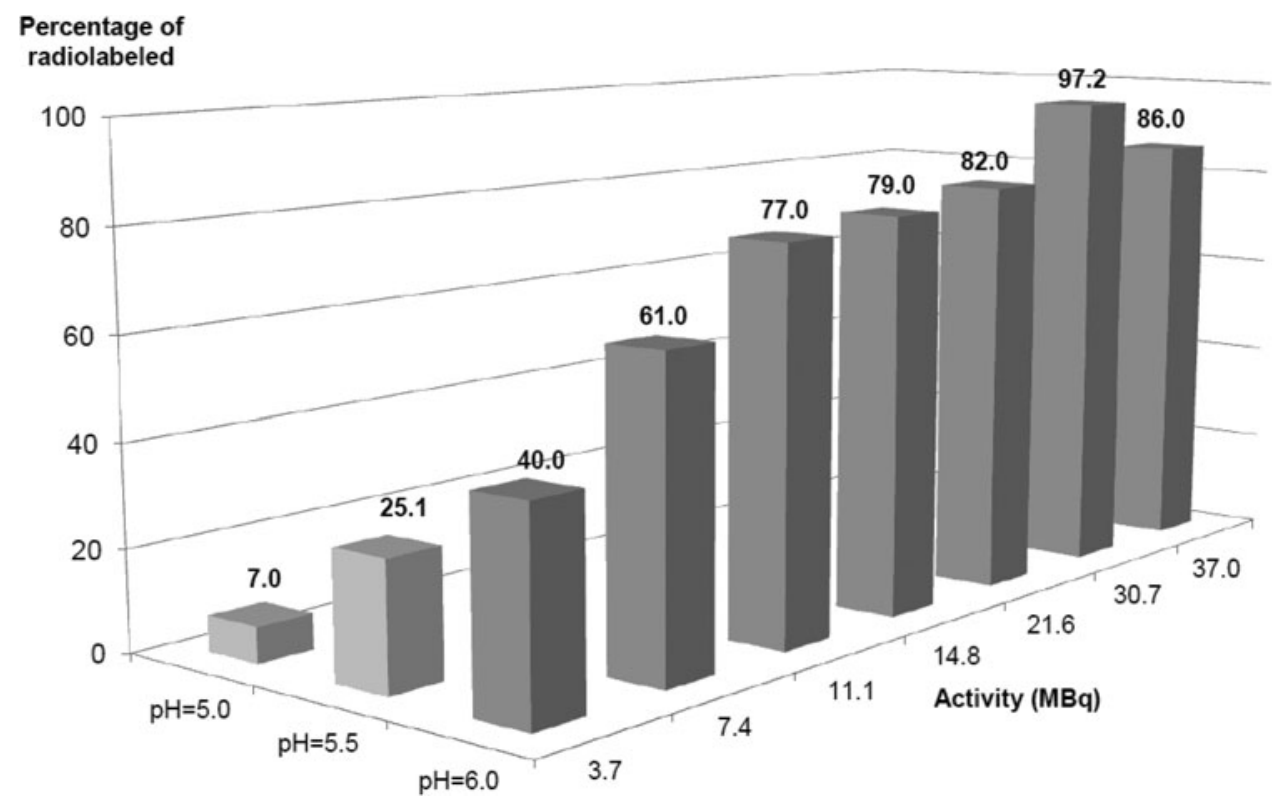

for 30 min (Supplementary Figs. S2 and S3). Probe stability on FBS resulted in high serum protein binding, $>90 \%$, and instability of free aptamer $(66 \%)$ was observed after $2 \mathrm{~h}$ of incubation (Supplementary Fig. S4). Similar results were obtained in the authors' previous reports. ${ }^{16}$

The PTK7 receptor affinity studies (Fig. 4) for the radio probe showed a $K_{\mathrm{d}}$ value at the low nanomolar, that is, $0.019 \mathrm{nM}$, indicating the binding affinity was not modified.

Besides, radio probe binding studies on tumor cell lines were performed based on previous results. ${ }^{16,18}$ A signal increase was observed over time for A20 and good binding since the first time point, $30 \mathrm{~min}$, for A20/GFP tumor cell line, demonstrating the Sgc8-c-NOTA- ${ }^{67} \mathrm{Ga}$ binding capability (Fig. 4).

In addition, to demonstrate that their model was adequate in the proof-of-concept study, the authors demonstrated that A20/GFP cells are able to express PTK7, using flow cytometry to analyze the A20/GFP cells (Supplementary Fig. S5).

\section{In vivo characterization}

The pharmacokinetic analysis showed that Sgc8-cNOTA- ${ }^{67} \mathrm{Ga}$ followed a two-compartment pharmacokinetic disposition in the body, with elimination from the central compartment. All mass transferences were assumed to follow first-order kinetics. Renal clearance was estimated using urine data in a sequential manner (i.e., after estimating pharmacokinetic parameters for probe disposition in body using blood data). Complete results and goodness-of-fit plots are included in the Supplementary Data. The elimination clearance (CL), including renal and no renal elimination pathways, was estimated to be $1.26 \mathrm{~mL} / \mathrm{h}$, the volumes of distribution for the central $(\mathrm{Vc})$ and peripheral compartments were 7.39 and $11.4 \mathrm{~L}$, respectively, and the distribution clearance (Q) was $1.22 \mathrm{~mL} / \mathrm{h}$. Renal clearance (CLr) was estimated at $0.172 \mathrm{~mL} / \mathrm{h}$. Interindividual variability was found significant for Vc $(\mathrm{CV}=19.7 \%)$, Q $(67.0 \%)$, and CLr $(45.9 \%)$. Using these primary pharmacokinetic parameters, a
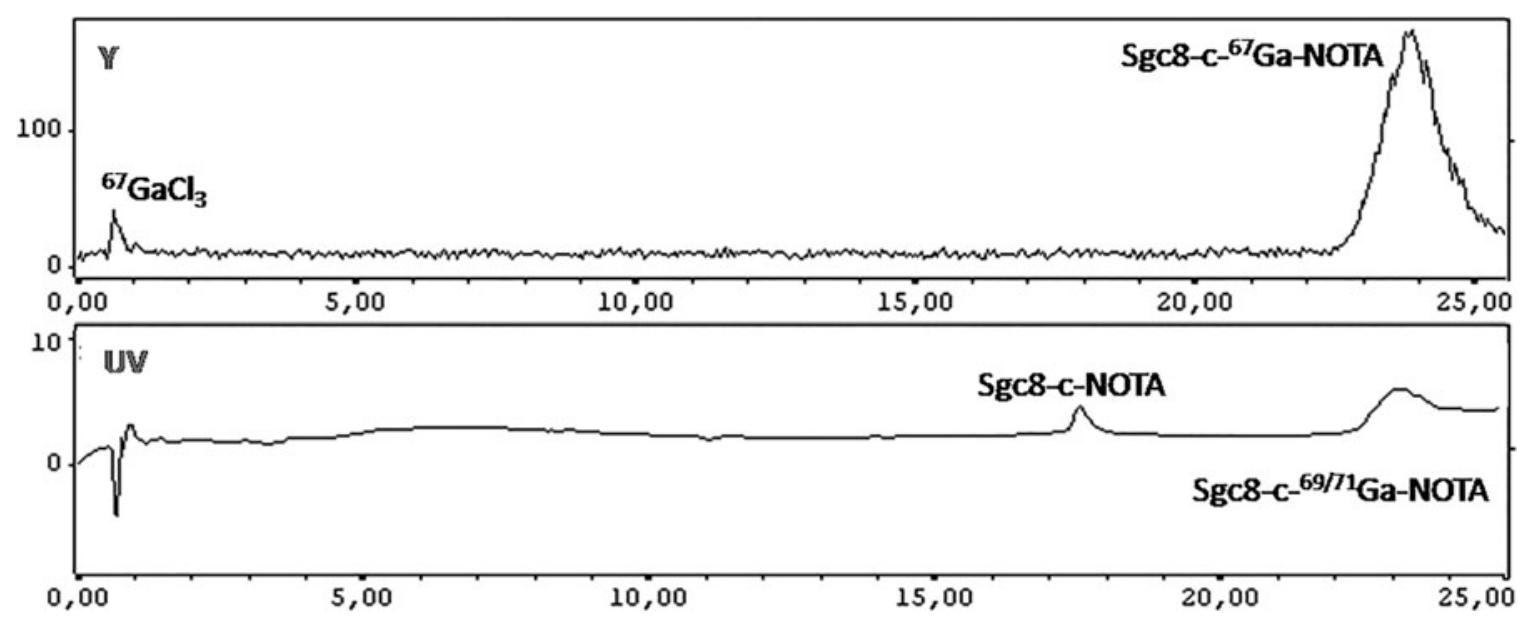

FIG. 3. RP-HPLC analysis ( $\gamma$-radiodetection, up, and UV detection at $260 \mathrm{~nm}$, down) to assess the identity of the Sgc8-cNOTA- ${ }^{67} \mathrm{Ga}$. RP-HPLC, reverse-phase high-performance liquid chromatography; UV, ultraviolet. 

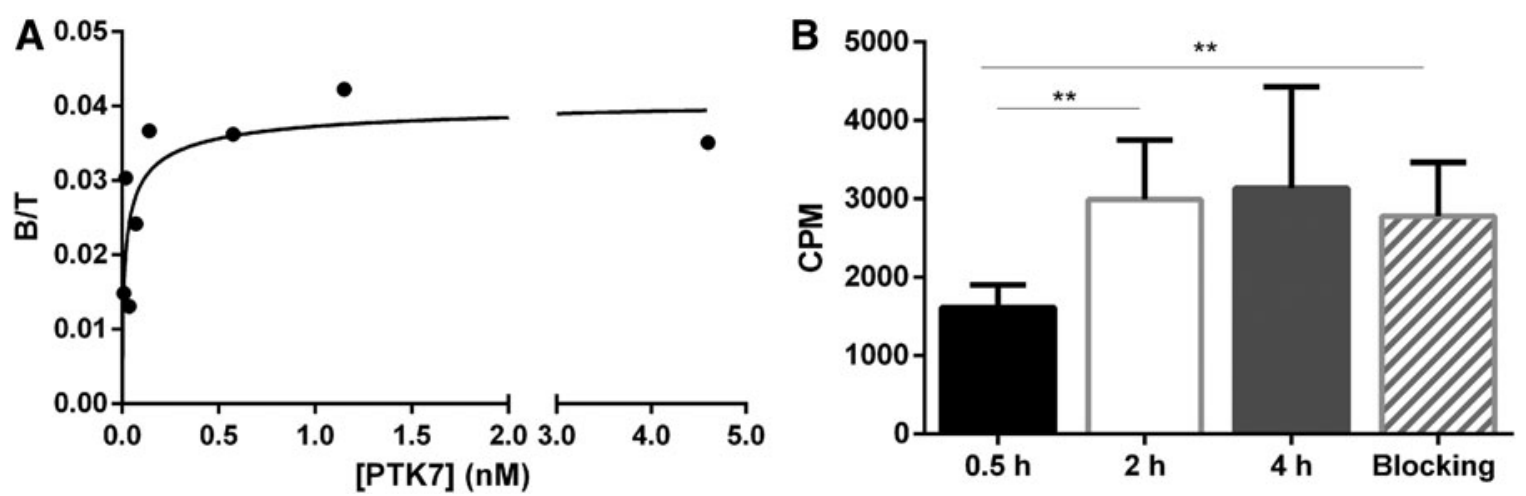

FIG. 4. Binding capacity. (A) PTK7-maximum binding capacity assay performed with Sgc8-c-NOTA- ${ }^{67}$ Ga. (B) Binding of Sgc8-c-NOTA- ${ }^{67}$ Ga to A20 cell line. ${ }^{* *} p<0.01$ (Student's $t$-test).

volume of distribution at steady state (Vss) of $18.8 \mathrm{~mL}$ and an elimination half-life of $15.1 \mathrm{~h}$ were obtained. Also, renal elimination accounted for $14.1 \%$ of the administered dosage (Supplementary Fig. S6).

The biodistribution of Sgc8-c-NOTA- ${ }^{67} \mathrm{Ga}$ in A20/GFP tumor-bearing BALB-c mice is shown in Figure 5. Low blood values were observed at all assayed time points. After $30 \mathrm{~min}$ postinjection, a value of $12.90 \pm 2.06 \% \mathrm{ID} / \mathrm{g}$ was observed, which decreased over time to $2.96 \pm 0.49 \% \mathrm{ID} / \mathrm{g}$ at $48 \mathrm{~h}$ postinjection. The hepatic activity levels remained constant along the assay, close to $20 \% \mathrm{ID} / \mathrm{g}$, in agreement with hepatobiliary metabolism. In addition, the bone radioactivity did not increase significantly with time, indicating coordinated gallium stability with similar behavior to that previously reported for Sgc8-c-DOTA- ${ }^{67} \mathrm{Ga} .{ }^{16}$ Bone radioactivity reached the maximum value at $24 \mathrm{~h}$ postinjection $\left(7.99 \pm 1.00 \% \mathrm{ID} / \mathrm{g}\right.$ for Sgc8-c-NOTA- $\left.{ }^{67} \mathrm{Ga}\right)$, with no affect on the quality of the images. It could be the result of some level of gallium release from Sgc8-c-NOTA- ${ }^{67} \mathrm{Ga}$ under physiological conditions. The urine activity slightly increased up to $24 \mathrm{~h}$.

The tumor uptake increased with the time being significantly higher since $48 \mathrm{~h}$ postinjection $(29.87 \pm 0.85 \% \mathrm{ID} / \mathrm{g})$ (Supplementary Fig. S7). In vivo blocking studies with Sgc8-c- $\mathrm{NH}_{2}$ showed, a statistically nonsignificant, decrease in tumor signal (Fig. 5). There was no significant uptake in the other no-target organs.
The blood and muscle ID/g activities decreased with time, resulting in better tumor/no-target ratios with time, reaching tumor/blood ratios higher than 7 and tumor/muscle ratios higher than 20, $24 \mathrm{~h}$ postinjection, for both ratios (Fig. 6).

In vivo imaging results showed consistent abdominal signal and high tumor uptake, which is mainly in the $24-\mathrm{h}$ time point (Fig. 7).

\section{Discussion}

Aptamers are a versatile tool in biotechnology field. Sharing characteristics of peptides and antibodies, aptamers have advantages due to their easy synthesis and facile chemical modification. ${ }^{18}$ These biomolecules can be modified to be functional for diverse approaches. In addition, their charge and low molecular weight result in a fast tissue penetration and clearance. Thus, aptamers have properties that make them ideal for theranostics purposes. Theranostics allow imaging visualization and treatment with the same probe, enabling personalized therapy. ${ }^{28}$

Previous studies showed Sgc8-c as a promising aptamer. ${ }^{18,29}$ This work studied an Sgc8-c derivative that was performed and radiolabeled with ${ }^{67} \mathrm{Ga}$. The NOTA conjugation was performed because of good properties as bifunctional chelator for $\mathrm{M}^{3+},{ }^{30,31}$ as ${ }^{177} \mathrm{Lu},{ }^{90} \mathrm{Y},{ }^{213} \mathrm{Bi}$, and ${ }^{67} \mathrm{Ga}$, which is a $\gamma$ emitting radionuclide $\left(t_{1 / 2}=78 \mathrm{~h}\right)$ used to perform imaging over days. ${ }^{64} \mathrm{Cu}$, a $\beta$ and positron emitter

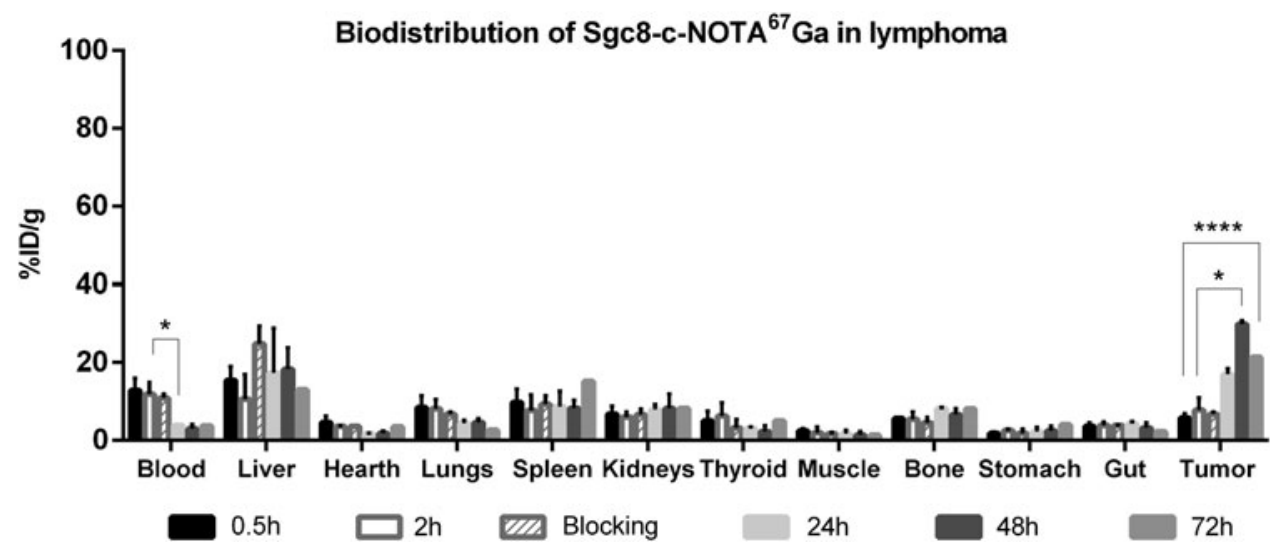

FIG. 5. Biodistribution. Results for Sgc8-cNOTA $-{ }^{67} \mathrm{Ga}$ at $0.5,2,24$, 48 , and $72 \mathrm{~h}$ postinjection in A20/GFP tumorbearing BALB-c mice. $* * * * p<0.0001, * p<0.05$ (Student's $t$-test). GFP, green fluorescent protein. 


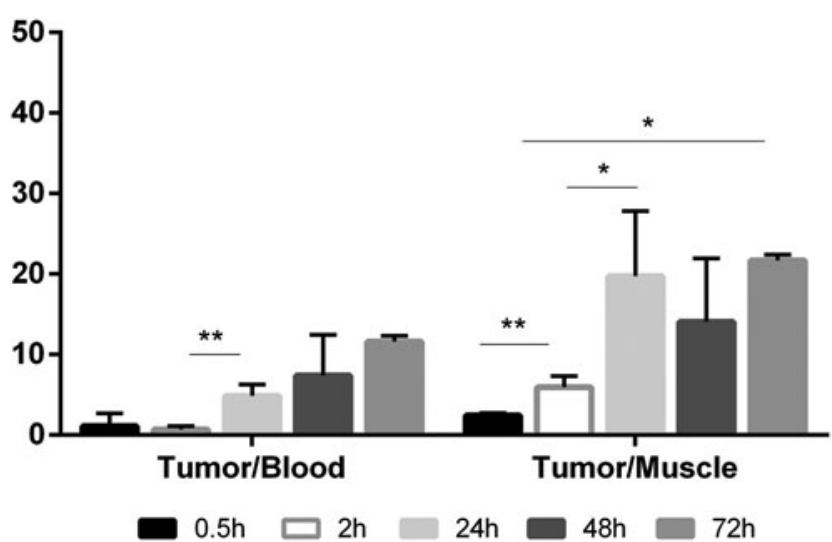

FIG. 6. Tumor/blood and tumor/muscle ratios. $* * p<0.01$, $* p<0.05$ (Student's $t$-test).

radionuclide, has also been chelated by NOTA and widely studied for positron emission tomography and theranostics. $^{32}$ Previous studies, ${ }^{33,34}$ together with the authors' results showed only one peak in the HPLC-analysis and the incorporation of only one gallium atom per molecule of aptamer, according to MS results, and, therefore, allowed us to propose the Ga coordination in the NOTA cage (Fig. 1).

Radiochemical purity and specific activity were evaluated for Sgc8-c-NOTA- ${ }^{67} \mathrm{Ga}$, and optimization procedure showed that the buffer with an adequate $\mathrm{pH}$ plays a relevant role in the labeling procedure. The rdl was over $95 \%$ and the identity of radio probe was verified using the chromatographic properties of the stable complex, Sgc8-c-NOTA- ${ }^{69 / 71} \mathrm{Ga}$. The Sgc8-c-NOTA- $-{ }^{67} \mathrm{Ga}$ distribution coefficient $\left(\log \mathrm{D}_{7.5}\right)$ was
$-2.41 \pm 0.11$, being a little more hydrophilic than the DOTA analogue previously described by the authors' group, that is, $\log \mathrm{D}_{7.5}=-1.87 \pm 0.05$ for Sgc8-c-DOTA- ${ }^{67} \mathrm{Ga} .{ }^{16}$ This change could modulate the in vivo behavior according to their aims. The radiolabeled probe showed high stability in buffer reaction and high binding serum protein.

Affinity studies showed a $K_{\mathrm{d}}$ value at low nanomolar for the PTK7-receptor, consistent with the data against entire CCRF-CEM cells, that is, $K_{\mathrm{d}} 0.80 \mathrm{nM}$, obtained by Tan et al. ${ }^{14}$ The radiolabeled probe was evaluated in vitro and in vivo in a lymphoma model.

The animal studies resulted in a two-compartment pharmacokinetic disposition in the body of the Sgc8-cNOTA- ${ }^{67} \mathrm{Ga}$. The biodistribution of Sgc8-c-NOTA- ${ }^{67} \mathrm{Ga}$ in A20/GFP tumor-bearing BALB-c mice resulted in no significant uptake in the no-target organs. Compared to the ${ }^{67} \mathrm{Ga}-$ radiolabeled analogue prepared using Sgc8-c-DOTA, ${ }^{16}$ the authors could state that the change in the NOTA-connector/ chelator did not modify the in vivo biodistribution as they expected, based on $\log \mathrm{D}$ values. ${ }^{16}$

Tumor uptake increased with the time being significantly higher for $48 \mathrm{~h}$ postinjection (Supplementary Fig. S7). However, the blood and muscle ID/g activities decreased with time, resulting in higher tumor/no-target ratios $24 \mathrm{~h}$ postinjection for both ratios (Fig. 6). These results were compared with a blocking control group, obtaining nonsignificant tumor uptake decrease. High serum protein binding can result in lack of blocking. In addition, high receptor expression could impose a low blocking result. The competition could be improved with changes in injected doses of radio probe and blocking agent in the future.

Biodistribution results were consistent with the in vivo images using multimodal tools. The authors observed high
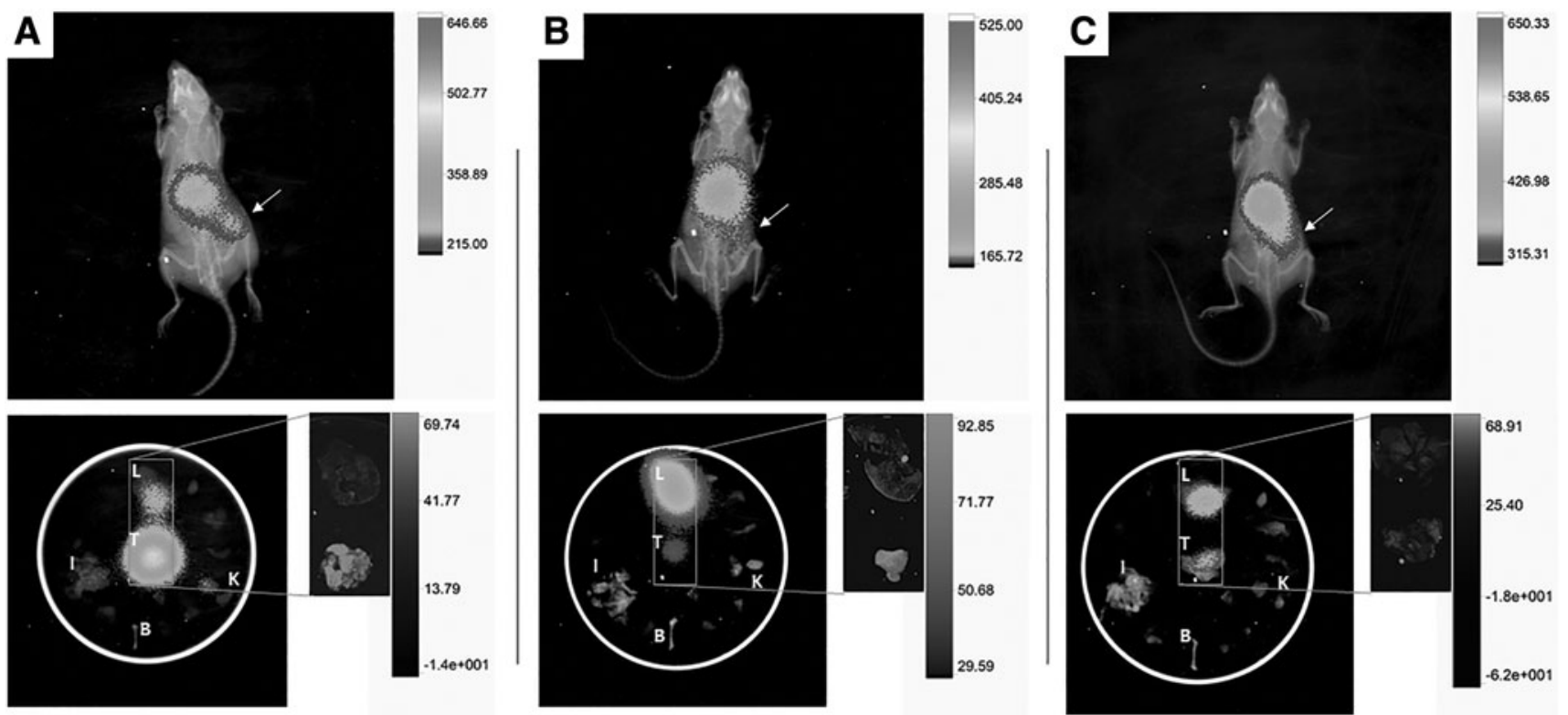

FIG. 7. Examples of multimodal images of radio probe-injected animals. Images were acquired at $24 \mathrm{~h}(\mathbf{A}), 48 \mathrm{~h}(\mathbf{B})$, and $72 \mathrm{~h}(\mathbf{C})$ postinjection. Up: whole animal X-rays and $\gamma$ live images; tumor is arrow indicated. Down: ex vivo X-ray and $\gamma$ images, including T: tumor, L: liver, I: intestine, B: bone, and K: kidneys. Ex vivo images also include lungs, blood, heart, muscle, stomach, thyroid, and spleen. Inset: additional A20/GFP cells fluorescence acquisition is highlighted in orange scale for liver and tumor. GFP, green fluorescent protein. 
tumor uptake and an abdominal signal at $24 \mathrm{~h}$ postinjection (Fig. 7, up). In addition to the well-defined tumor image at $24 \mathrm{~h}$, at 48 and $72 \mathrm{~h}$, there were image-asymmetries that are evidence of possible tumor presence, which was confirmed with co-localization studies (Fig. 7, down inset). When the organs were analyzed separately (ex vivo), it was possible to distinguish high tumor and liver signals (Fig. 7, down), which agreed with the biodistribution studies (Fig. 5). In some cases, the signals from the tumor were the highest. In addition, using the multimodal combination of X-ray, fluorescence, and $\gamma$, they could co-localize the fluorescent tumoral cells and the radio probe in the same location where the GFP was visualized in the studied tumors (Fig. 7, inset), being an adequate positive control of specific interaction.

Furthermore, additional investigation is needed to enhance tumor retention over time. However, tumor uptake could be improved with doses and time acquisition adjustments. In addition, studies with human tumor xenograft murine model should be done as previous translational step. Finally, the high tumor/no-target ratios of Sgc8-c-NOTA- ${ }^{67}$ Ga highlight its potential as theranostic agent.

\section{Conclusions}

In this study, the authors observed considerable uptake of the radio probe in the A20-tumor since the initial time point, increasing after $24 \mathrm{~h}$, with the concomitant signal decreasing in the blood and muscles. Consequently, target/no-target signal ratios were very high for this mouse model and this effect improved image contrast. Thus, they confirmed the utility of the Sgc8-c aptamer as theranostic agent for hemato-oncological malignancies.

\section{Acknowledgments}

The authors specially thank Thais Bascuas for her assistance in the A20/GFP construction. ANII is grateful (POS_NAC_2017_1_140364). All coauthors have reviewed and approved the article before submission.

\section{Authors' Contributions}

E.S. is a $\mathrm{PhD}$ student and she worked in all of the experimental activities. J.B. contributed in radiolabeled and quality control experiments. M.I. contributed in pharmacokinetics analyses, discussion of the results, and writing the article. M.F. contributed in all of the animal experiments. P.C. contributed in the discussion of the results. M.M. contributed in the cell studies, discussion of the results, and writing the article. H.C. contributed in the planning of the activities, discussion of the results, and writing the article. V.C. contributed in the planning of the activities, discussion of the results, and writing the article.

\section{Disclosure Statement}

There are no existing financial conflicts.

\section{Funding Information}

This research was supported by a grant from the MEC, FVF_24_Calzada (Montevideo, Uruguay).

\section{Supplementary Material}

Supplementary Table S1 Supplementary Table S2 Supplementary Table S3 Supplementary Table S4 Supplementary Table S5 Supplementary Figure S1 Supplementary Figure S2 Supplementary Figure S3 Supplementary Figure S4 Supplementary Figure S5 Supplementary Figure S6 Supplementary Figure S7

\section{References}

1. Bayat P, Nosrati R, Alibolandi M, et al. SELEX methods on the road to protein targeting with nucleic acid aptamers. Biochimie 2018;154:132.

2. Zhou J, Satheesan S, Li H, et al. Cell-specific RNA aptamer against human CCR5 specifically targets HIV-1 susceptible cells and inhibits HIV-1 infectivity. Chem Biol 2015;22:379.

3. Wang CY, Lin BL, Chen $\mathrm{CH}$. An aptamer targeting shared tumor-specific peptide antigen of MAGE-A3 in multiple cancers. Int J Oncol 2016;138:918.

4. Wolter AC, Weickhmann AK, Nasiri AH, et al. A stably protonated adenine nucleotide with a highly shifted $\mathrm{pKa}$ value stabilizes the tertiary structure of a GTP-binding RNA aptamer. Angew Chem Int Ed 2017;56:401.

5. Huang Y, Chen X, Duan N, et al. Selection and characterization of DNA aptamers against Staphylococcus aureus enterotoxin C1. Food Chem 2015;166:623.

6. Ramezani M, Abnous K, Taghdisi SM. Optical and electrochemical aptasensors for sensitive detection of streptomycin in blood serum and milk. Methods Mol Biol 2017; 1572:403.

7. Alshaer W, Hillaireau H, Fattal E. Aptamer-guided nanomedicines for anticancer drug delivery. Adv Drug Deliv Rev 2018;134:122.

8. Ruiz Ciancio D, Vargas MR, Thiel WH, et al. Aptamers as diagnostic tools in cancer. Pharmaceuticals 2018;11:E86.

9. Yang S, Li H, Xu L, et al. Oligonucleotide aptamermediated precision therapy of hematological malignancies. Mol Ther Nucleic Acids 2018;13:164.

10. Wu X, Liang H, Tan Y, et al. Cell-SELEX aptamer for highly specific radionuclide molecular imaging of glioblastoma in vivo. PLoS One 2014;9:e90752.

11. Ni S, Yao H, Wang L, et al. Chemical modifications of nucleic acid aptamers for therapeutic purposes. Int $\mathrm{J}$ Mol Sci 2017;18:E1683.

12. Healy JM, Lewis SD, Kurz M, et al. Pharmacokinetics and biodistribution of novel aptamer compositions. Pharm Res 2004;21:2234.

13. Shangguan D, Li Y, Tang Z, et al. Aptamers evolved from live cells as effective molecular probes for cancer study. Proc Natl Acad Sci U S A 2006;103:11838.

14. Prebet T, Lhoumeau AC, Arnoulet C, et al. The cell polarity PTK7 receptor acts as a modulator of the chemotherapeutic response in acute myeloid leukemia and impairs clinical outcome. Blood 2010;116:2315.

15. McCarten KM, Nadel HR, Shulkin BL, et al. Imaging for diagnosis, staging and response assessment of Hodgkin lymphoma and non-Hodgkin lymphoma. Pediatr Radiol 2019;49:1545. 
16. Calzada V, Báez J, Sicco E, et al. Preliminary in vivo characterization of a theranostic aptamer: Sgc8-cDOTA- ${ }^{67}$ Ga. Aptamers 2017;1:19-27.

17. Notni J, Pohle K, Wester HJ. Comparative gallium-68 labeling of TRAP-, NOTA-, and DOTA-peptides: Practical consequences for the future of gallium-68-PET. EJNMMI Res 2012;2:28.

18. Calzada V, Moreno M, Newton J, et al. Development of new PTK7-targeting aptamer-fluorescent and -radiolabelled probes for evaluation as molecular imaging agents: Lymphoma and melanoma in vivo proof of concept. Bioorg Med Chem 2017;25:1163.

19. Sicco E, Báez J, Margenat J, et al. Derivatizations of Sgc8-c aptamer to prepare metallic radiopharmaceuticals as imaging diagnostic agents: Syntheses, isolations, and physicochemical characterizations. Chem Biol Drug Des 2018;91:747.

20. Kalil RA, Teixeira LA, Mastalir ET, et al. Experimental model of gene transfection in healthy canine myocardium: Perspectives of gene therapy for ischemic heart disease. Arq Bras Cardiol 2002;79:223.

21. Jacobson O, Weiss ID, Wang L, et al. Labeled singlestranded DNA aptamer for PET imaging of protein tyrosine kinase-7 expression. J Nucl Med 2015;56:1780.

22. Lindmo T, Bunn PA. Determination of the true immunoreactive fraction of monoclonal antibodies after radiolabeling. Methods Enzymol 1986;121:678.

23. Beal S, Sheiner LB, Boeckmann A, et al. NONMEM User's Guides (1989-2009). Ellicott City, MD: Icon Development Solutions, 2009.

24. Bascuas T, Moreno M, Mónaco M, et al. A novel nonHodgkin lymphoma murine model closer to the standard clinical scenario. J Transl Med 2016;14:323.

25. Gijs M, Dammicco S, Warnier C, et al. Gallium-68-labelled NOTA-oligonucleotides: An optimized method for their preparation. J Label Compd Radiopharm 2016;59:63.
26. Kryza D, Debordeaux F, Azéma L, et al. Ex vivo and in vivo imaging and biodistribution of aptamers targeting the human matrix metalloprotease-9 in melanomas. PLoS One 2016;11:e0149387.

27. Velikyan I, Beyer GJ, Bergström-Pettermann E, et al. The importance of high specific radioactivity in the performance of ${ }^{68} \mathrm{Ga}$-labeled peptide. Nucl Med Biol 2008;35: 529.

28. Drude N, Tienken L, Mottaghy FM. Theranostic and nanotheranostic probes in nuclear medicine. Methods 2017; 130:14.

29. Duan S, Yu Y, Lai C, et al. Vincristine-loaded and Sgc8modified liposome as a potential targeted drug delivery system for treating acute lymphoblastic leukemia. J Biomed Nanotechnol 2018;14:910.

30. Kretschy D, Koellensperger G, Hann S. Stability assessment of different chelating moieties used for elemental labeling of bio-molecules. Metallomics 2011;3:1304.

31. Kang L, Rosenkrans ZT, Cai W. ${ }^{64} \mathrm{Cu}$-Labeled aptamers for tumor-targeted radionuclide delivery. Methods Mol Biol 2019;1974:223.

32. Woo SK, Jang SJ, Seo MJ, et al. Development of ${ }^{64} \mathrm{Cu}-$ NOTA-trastuzumab for HER2 targeting: A radiopharmaceutical with improved pharmacokinetics for human studies. J Nucl Med 2019;60:26.

33. Chakraborty S, Goswami D, Chakravarty R, et al Syntheses and evaluation of ${ }^{68} \mathrm{Ga}$ - and ${ }^{153} \mathrm{Sm}$-labeled DOTA-conjugated bisphosphonate ligand for potential use in detection of skeletal metastases and management of pain arising from skeletal metastases. Chem Biol Drug Des 2018;92:1618.

34. Holub J, Meckel M, Kubíček V, et al. Gallium(III) complexes of NOTA-bis(phosphonate) conjugates as PET radiotracers for bone imaging. Contrast Media Mol Imaging 2015;10:122. 